Article

\title{
Revisiting the 1D and 2D Laplace Transforms
}

\author{
Manuel Duarte Ortigueira ${ }^{1, *, t, \ddagger(D)}$ and José Tenreiro Machado ${ }^{2, t, f(\mathbb{D})}$ \\ 1 CTS-UNINOVA and DEE of NOVA School of Science and Technology, 2829-516 Caparica, Portugal \\ 2 Institute of Engineering, Polytechnic of Porto, Department of Electrical Engineering, \\ 431 4249-015 Porto, Portugal; jtm@isep.ipp.pt \\ * Correspondence: mdo@fct.unl.pt \\ + Current address: Campus of NOVA School of Science and Technology, Quinta da Torre, \\ 2829-516 Caparica, Portugal. \\ $\ddagger$ These authors contributed equally to this work.
}

Received: 13 July 2020; Accepted: 6 August 2020; Published: 10 August 2020

\begin{abstract}
The paper reviews the unilateral and bilateral, one- and two-dimensional Laplace transforms. The unilateral and bilateral Laplace transforms are compared in the one-dimensional case, leading to the formulation of the initial-condition theorem. This problem is solved with all generality in the one- and two-dimensional cases with the bilateral Laplace transform. The case of fractional-order systems is also included. General two-dimensional linear systems are introduced and the corresponding transfer function is defined.
\end{abstract}

Keywords: laplace transform; two-dimensional laplace transform; initial-conditions; two-dimensional linear systems

MSC: 26A33

\section{Introduction}

The importance of the Laplace transform (LT) in many mathematical and scientific areas is unquestionable making it one of the most useful mathematical tools in applications, as Circuit Theory [1-3], Signals and Systems [4-6], Control [7,8], Probability and Statistics [9-12], Bioengineering [13], Mechanical Engineering [14], and others [15-17]. Probably, the main motivation for this interest was Heaviside's operational calculus [18-26], due to the role played by the LT in its validation. The unilateral version of the LT has gained preference in a large part of the applications, but the bilateral LT has increased its influence in the last 40 years in parallel with the development of the area of signals and systems [4-6]. The unique advantage of the unilateral (ULT) over the bilateral (BLT) version of the LT is its ability of introducing the initial-conditions in differential equations. However, if this advantage can be overcome by an appropriate strategy based on the BLT, then there is no other particular reason for using the ULT and the BLT is the most adequate tool to use in practical applications.

In this survey paper, we compare the two versions of the LT by reviewing, step by step, the construction of these transforms in the one-dimensional (1D) case. We introduce a methodoly for solving the initial-condition problem without requiring the adoption of the ULT. Moreover, during the process we consider a new general formulation including fractional order differential equations.

The formulation of the two-dimensional LT (2D-LT) is then essayed by following the steps exercised for the 1D-BLT and their properties of the 2D-LT are discussed. We mainly focus on the less known two-sided transform. Additionally, 2D differential equations representing linear systems and the initial conditions for the 2D linear systems are also analyzed. 
The paper is outlined as follows. Section 2 presents a brief historical review regarding the development of the ULT and BLT. Furthermore, in the sequel of their introduction, we include the description and comparison of their properties. The initial-condition problem is studied and a general solution is proposed based on the BLT. Section 3 addresses the 2D-BLT and its properties. Section 4 discusses the definition of 2D linear systems, and the concept of 2D fractional derivatives. Moreover, the $2 \mathrm{D}$ initial-condition problem is studied and a solution is proposed. Finally, Section 5 draws the conclusions.

\section{Continuous-Time Laplace Transforms}

\subsection{One-Sided Laplace Transform}

The one-sided or unilateral Laplace transform (ULT) was conceived by Euler (1763) as a tool for solving some differential equations [27-29]. Nonetheless, the first real use of such operator was brought about by Laplace in the scope of several studies (1779-1812), with main focus on Probability Theory. This transform was studied and used by several other mathematicians like Lagrange, Lacroix, Fourier, Cauchy, Abel, and Liouville [28,29]. It is interesting to point out that the first major application of the 2D-LT was developed by Cauchy [28]. It is also important to highlight the work of Liouville because he used the ULT as a base for his definitions of fractional derivatives [30-34]. However, Liouville had a limitation in his calculations since the inverse LT was not known at that time. We do not mean that formulae similar to what is known today as inversion integral were not yet established. In fact, Petzval and Riemann presented an integral formulation very similar to the so-called Bromwich inversion formula, but apparently they were not aware that it was really a LT inverse [28]. This fact was discovered in 1926, in the sequel of Bromwich's work, by P. Lévy [23]. In the last two decades of the XIX century, the ULT was rediscovered by Poincare [29] and attracted the interest of other mathematicians, being important to refer Pincherle that formulated his transform in the complex plane (1887) [29]. Picard proposed a 2D-LT to solve partial differential Equations (1888). Soon later, the unicity and convergence problems associated to the LT were considered by Lerch (1892) [29]. We find the important works of Mellin in the last decade of the XIX century, addressing the relation between the Laplace and the Mellin transforms. Meanwhile, the Heaviside's Operational Calculus (1892-1912) [18-22] created a great stir, but in view of the quality and importance of its results, there was a need for a solid mathematical justification [25]. To achieve such an objective several researchers invested considerable efforts. The most interesting were based on the LT. We highlight the works of Bromwich, with his integral formulation, Lévy, as referred previously, Carson [1,2,35], with the ULT, Van Der Pol [36], with the two-sided LT, and mainly Doetsch [37] with a very critical view of Heaviside's procedures. Carson and Van der Pol used the " $p$-multiplied" LT given by $F(p)=$ $p \int f(t) e^{-p t} d t$. In 1937, Doetsch published a very rigorous, coherent and clear formulation of the ULT and its applications, mainly to differential equations $[37,38]$. This book became very popular and somehow imposed the ULT as a "standard" tool for solving constant coefficient differential equations. We can say that it is "the" LT for most mathematicians and scientists, even if it poses difficulties in the initial valued problems [39-41].

\subsection{The Bilateral Transform}

The two-sided or bilateral Laplace transform (BLT) emerged and was developed in an independent way, and is mainly the result of the efforts of Van der Pol and Bremmer [36]. Their work has several interesting features:

1. Creates a unified framework which includes the ULT and the Fourier transform (FT) as particular cases,

2. Gives a justification for Heaviside's operations, while giving insights into generalizations to the fractional case [40],

3. Introduces the 2D-LT theory. 
However, their formulation had a drawback: they used a " $p$-multiplied" version [36]. It is important to refer two other contributions, one of Paley and Wiener, on the FT in the complex plane [42], and another by Widder, that considered both the ULT and BLT in the framework of the Stieltjes integral [43]. These works were fundamental in asserting the BLT as an independent operator alternative to the ULT. The two contributions removed the multiplicative $p$ factor used previously by Carson, Van der Pol, and Bremmer.

We can say that the BLT has several advantages over the ULT, namely [6]:

1. It is more general than the ULT, which is a particular case of the first,

2. Offers insight into the nature of system characteristics such as stability, causality, and frequency response,

3. Allows the study not only of causal systems, but also of the anti-causal and acausal,

4. Includes the FT as particular case.

5. The class of functions to which the BLT can be applied is much larger than the ones that have BLT,

6. Some properties, e.g., derivative and translation, become simplified, when compared with the ones of ULT.

\subsection{Constructing the BLT}

The starting point to introduce the BLT is the Fourier identity [36]

$$
h(t)=\frac{1}{2 \pi} \int_{-\infty}^{\infty} d \omega \mathrm{e}^{i \omega t} \int_{-\infty}^{\infty} d \tau \mathrm{e}^{-i \omega \tau} h(\tau),
$$

where $i=\sqrt{-1}$.

In applications, it is usual to decompose the relation (1) into

$$
\begin{gathered}
\mathcal{F}[h(t)]=H(i \omega)=\int_{-\infty}^{\infty} h(t) e^{-i \omega t} \mathrm{~d} t, \omega \in \mathbb{R}, \\
h(t)=\mathcal{F}^{-1}[X(i \omega)]=\frac{1}{2 \pi} \int_{-\infty}^{\infty} H(i \omega) e^{i \omega t} \mathrm{~d} \omega, t \in \mathbb{R},
\end{gathered}
$$

which are called analysis equation and synthesis equation, respectively. The integration must be taken in the sense of the Cauchy principal value. Relation (3) has an interesting interpretation that justifies the name: a continuous-time function can be expressed as a continuous overlap of elemental sisoids with infinitesimal complex amplitudes, $\frac{1}{2 \pi} X(\omega) d \omega$, and frequencies, $\left.\omega \in\right]-\infty,+\infty$ [ infinitely close. The function $H(i \omega)$ in (42) is the (direct) FT, while $h(t)$ given by (3) is the inverse FT. About the existence of the FT (2) we can say that if $h(t)$ is piecewise continuous, with bounded variation, and absolutely integrable (AI), then the FT exists and the convergence of the integral in (1) is uniform on $\omega \in]-\infty,+\infty[[4,5]$.

In the scope of the introduction of the BLT, we take (3) and rewrite it as an integral along the imaginary axis

$$
h(t)=\frac{1}{2 \pi i} \int_{-i \infty}^{i \infty} H(s) e^{s t} \mathrm{~d} s, t \in \mathbb{R},
$$

and therefore we can generalize it by integrating along any vertical straight line contained in a strip where $H(s)$ is analytic [42]. 
Definition 1. We define the inverse LT by

$$
h(t)=\mathcal{L}^{-1} F(s)=\frac{1}{2 \pi i} \int_{a-i \infty}^{a+i \infty} H(s) e^{s t} \mathrm{~d} s, t \in \mathbb{R},
$$

where $a \in \mathbb{R}$ is called abscissa of convergence. The right hand side represents the Bromwich integral. In the following we may denote by $\gamma$ the integration path.

If we interpret the integral in Equation (5) as previously for expression (3), then we can say that a function $h(t)$ can be considered as a superposition of elemental complex exponentials, $h(t)=$ $\frac{1}{2 \pi i} \int_{\gamma} F(s) d s \cdot e^{s t}$. This result is very interesting because the exponentials are the eigenfunctions of the linear systems.

Definition 2. The direct LT, corresponding to the synthesis Equation (5), is obtained from (2) by the substitution s for $i \omega$ :

$$
\mathcal{L}[h(t)]=H(s)=\int_{-\infty}^{\infty} h(t) e^{-s t} \mathrm{~d} t, s \in \mathbb{C}
$$

We can obtain existence conditions for the BLT, from those of the FT $[4,36,44]$.

Let $h(t)$ be a function piecewise continuous, with bounded variation, locally integrable (in the sense that the function is absolutely integrable in any real interval $[a, b]$, so that $\left.\int_{a}^{b}|h(t)| \mathrm{d} t<\infty\right)$, and of exponential order, then there exists the BLT of $h(t)$. Loosely speaking, a function of exponential order is the one that does not "grow faster" than given exponentials, as $t \rightarrow \pm \infty$. This means two things. First, that there are real constants $A, a>0$ such that $|h(t)|<A \cdot e^{a t}$, when $t$ is large and negative (say, for $t<t_{1} \in \mathbb{R}$ ). Second, that there are real constants $B, b>0$ such that $|h(t)|<B \cdot e^{b t}$, when $t$ is large (say, for $t>t_{2} \in \mathbb{R}$ ). It also has to be true that $b<a$.

Under these conditions, the integral in (6) converges absolutely and uniformly in a vertical strip in the complex plane defined by $b<\operatorname{Re}(s)<a$, where $H(s)$ is analytic. This strip is called region of convergence (ROC), and the values of the constants $a$ and $b$ are the abscissas of convergence. It is possible to show that the inversion integral (5) converges to the half sum of the lateral values, that is, $\frac{1}{2}\left[h\left(t^{+}\right)+h\left(t^{-}\right)\right]$, for any $t \in \mathbb{R}[36]$.

Remark 1. The most usual procedure for computing $H(s)$ is based on the decomposition of the integral (6) in two parts:

$$
\mathcal{L}[h(t)]=\int_{-\infty}^{\infty} h(t) e^{-s t} \mathrm{~d} t=\int_{-\infty}^{0} h(t) e^{-s t} \mathrm{~d} t+\int_{0}^{\infty} h(t) e^{-s t} \mathrm{~d} t
$$

Both integrals on the right hand side are one-sided LT. In particular, $\int_{0}^{\infty} h(t) e^{-s t} \mathrm{~d} t$ is the common LT and will be denoted by ULT (Section 2.4). It must be referred that the first and second transforms are convergent for $\operatorname{Re}(s)<a$ and $\operatorname{Re}(s)>b$, respectively. Therefore, there will exist the BLT of $h(t)$ if and only if $b<a$.

Since the Euler's introduction in1925, the ULT has been used without the inversion integral, but nowadays (5) is the base for solving some non elemental problems. This implies that it is advisable we show that (5) is really the inverse of (42).

Theorem 1. Under the existence conditions, expression (5) inverts (6), that is, we have:

$$
\frac{1}{2 \pi i} \int_{a-i \infty}^{a+i \infty} \int_{-\infty}^{\infty} h(\tau) e^{-s \tau} \mathrm{d} \tau e^{s t} \mathrm{~d} s=h(t), t \in \mathbb{R} .
$$


Proof. The inner integral converges absolutely and uniformly for any $s$ in the ROC. Therefore, we can interchange the operations of integration:

$$
\int_{-\infty}^{\infty} h(\tau) \frac{1}{2 \pi i} \int_{a-i \infty}^{a+i \infty} e^{-s \tau} e^{s t} \mathrm{~d} s \mathrm{~d} \tau
$$

Let us consider the inner integral. We can write it as:

$$
\frac{1}{2 \pi i} \int_{a-i \infty}^{a+i \infty} e^{s(t-\tau)} \mathrm{d} s=\frac{1}{2 \pi} e^{a(t-\tau)} \int_{-\infty}^{+\infty} e^{i \omega(t-\tau)} \mathrm{d} \omega=\delta(t-\tau)
$$

Attending to the properties of the delta distribution $\delta$, the convolution yields $h(t) * \delta(t)=h(t)$ (where $*$ denotes convolution and $\delta$ is the neutral element) and we obtain the result.

Remark 2. We can define different time functions $f(t)$ from the same $F(s)$, since we only have to choose different regions of analyticity for locating the integration path. For example, if $F(s)=\frac{s}{(s-1)(s+2)}$, then we can find three analyticity regions, $\operatorname{Re}(s)<-2,-2<\operatorname{Re}(s)<1, \operatorname{Re}(s)>1$, and consequently (5) defines three different functions.

Remark 3. Let $h(t)=0, t<T \in \mathbb{R}$ (called right signal). If we choose $t<t_{1}<0$, then $a=\infty$ and the $R O C$ is $\operatorname{Re}(s)>b$. Therefore, the ROC corresponding to a right signal is a right half plane. Similarly, if $h(t)=0, t>T \in \mathbb{R}$ (left signal), then the ROC is a left half plane.

The works of Paley and Wiener [42] aimed to generalize the FT and it is the reason why they did not consider the name BLT. In fact, they adopted "Fourier transform in the complex domain" and were paying attention to the behavior in the transform domain. They started from the Plancherel theorem, that generalizes the FT to functions in $L_{2}$ (squared integrable), for defining a transform coinciding with the BLT. However, instead of operational aspects they studied its convergence properties, from which we must refer [42]:

1. If the region of convergence of $F(s)$ includes the frontiers $b \leq \operatorname{Re}(s) \leq a$, then $F(s)$ is completely defined in that region by the values at that lines, $F(a+i t)$ and $F(b+i t), t \in \mathbb{R}$.

2. $F(s)$ is bounded in the strip $a+\epsilon \leq \operatorname{Re}(s) \leq b-\epsilon$, with $\epsilon>0$.

The BLT enjoys several properties, most of them easily deduced from the inversion integral. If $F(s)$ is the LT of $f(t)$, i.e., $\mathcal{L} f(t)=F(s)$, with a given region of convergence, $\mathcal{R}_{c}$, then the LT has the following properties. Note that in all the properties the ROC is not defined, since it depends on the function at hand, although the properties are general.

1. Linearity/homogeneity

This is obvious, if we consider other function $(\mathcal{L} g(t)=G(s))$

$$
\mathcal{L}[a f(t)+b g(t)]=a F(s)+b G(s), \quad s \in \mathcal{R}_{c} .
$$

2. Time derivative

This property depends on how we define derivative and its action over the exponential. As we are working on $\mathbb{R}$, we assume that the Grünwad-Letnikov, Liouville (regularized), or Liouville-Caputo derivatives are used, meaning that if $\alpha \in \mathbb{R}$, then $D^{\alpha} e^{s t}=s^{\alpha}$ for $\operatorname{Re}(s)>0$ or $\operatorname{Re}(s)<0$ depending on the causality of the derivative [45]. From (6) we obtain

$$
D^{\alpha} f(t)=\frac{1}{2 \pi i} \int_{\gamma} s^{\alpha} F(s) e^{s t} d s, \quad t \in \mathbb{R}
$$


from which we deduce that,

$$
\mathcal{L}\left[D^{\alpha} f(t)\right]=s^{\alpha} F(s)
$$

This property is valid for any $\alpha$ complex. The dual of this property reads

$$
\mathcal{L}\left[(-t)^{\alpha} f(t)\right]=F^{(\alpha)}(s) .
$$

This dual property creates a difficulty: the essential singularity at $t=0$.

3. Scale change

It is almost immediate with the substitution of $a t, a \in \mathbb{R}$, for $t$, into (5)

$$
\mathcal{L}[f(a t)]=\frac{1}{|a|} F(s / a) .
$$

4. Time reversion

$$
\mathcal{L}[f(-t)]=F(-s)
$$

This is a consequence of (13), obtained with $a=-1$.

5. Time shift

Let $a \in \mathbb{R}$. Then

$$
\mathcal{L}[f(t-a)]=e^{-a s} F(s) .
$$

6. Modulation

This is the dual of the previous property,

$$
\mathcal{L}\left[e^{a t} f(t)\right]=F(s-a) .
$$

Example 1. If $g(t)=f(t) \cdot \cos \left(\omega_{0} t\right)$, then $G(s)=\frac{1}{2} F\left(s-i \omega_{0}\right)+\frac{1}{2} F\left(s+i \omega_{0}\right)$.

7. Convolution

Define, with all the generality, the convolution between two functions $f(t)$ and $g(t)$ by:

$$
f(t) * g(t)=\int_{-\infty}^{\infty} f(\tau) g(t-\tau) \mathrm{d} \tau
$$

and insert expression (15) to get

$$
\begin{gathered}
f(t) * g(t)=\int_{-\infty}^{\infty} f(\tau) \frac{1}{2 \pi i} \int_{\gamma} G(s) e^{s t} e^{-s \tau} d s \mathrm{~d} \tau=\frac{1}{2 \pi i} \int_{\gamma} G(s)\left[\int_{-\infty}^{\infty} f(\tau) e^{-s \tau} \mathrm{d} \tau\right] d s, \\
F(s)=\mathcal{L} f(t)=\int_{-\infty}^{\infty} f(\tau) e^{-s \tau} \mathrm{d} \tau, \\
\mathcal{L}[f(t) * g(t)]=\mathcal{L}[f(t)] \mathcal{L}[g(t)] .
\end{gathered}
$$

8. The Abelian initial value Theorem

The initial value theorem (IVT) is a very important result when dealing with the LT $[38,43,46]$. This theorem relates the asymptotic behavior of a causal signal, $f(t)$, as $t \rightarrow 0^{+}$, to the asymptotic natureof its $\mathrm{LT}, F(s)=\mathcal{L}[f(t)]$, as $\sigma=\operatorname{Re}(s) \rightarrow \infty$. 
Theorem 2. Suppose that there are two real functions, $f(t)$ and $\varphi(t)$, defined and continuous in a given interval $0<t<T$, where $\varphi(t)$ is assumed to be positive. The LT of the two functions, denoted $F(s)$ and $\Phi(s)$, respectively, have the right half planes as ROC. Let $\sigma=\operatorname{Re}(s)$. We conclude that:

$$
f(t) \sim A \varphi(t) \text { for } t \rightarrow 0 \Longrightarrow F(\sigma) \sim A \Phi(\sigma), \text { for } \sigma \rightarrow \infty
$$

In particular, when $\varphi(t)=t^{\alpha} \varepsilon(t)$ we obtain the following result.

Theorem 3 (The initial-value Theorem). Assume that $f(t)$ is a causal signal such that in some neighborhood of the origin, it is a regular distribution, corresponding to an integrable function, and its LT is $F(s)$, with $\operatorname{ROC} \operatorname{Re}(s)>0$.

In addition, assume that there is a real number $\beta>-1$ such that $\lim _{t \rightarrow 0^{+}} f(t) t^{\beta}$ exists and is a finite complex value. Then

$$
\lim _{t \rightarrow 0^{+}} \frac{f(t)}{t^{\beta}}=\lim _{\sigma \rightarrow \infty} \frac{\sigma^{\beta+1} F(\sigma)}{\Gamma(\beta+1)} .
$$

The proof of this Theorem can be found in [46] (Section 8.6, pages 243-248).

9. The final value Theorem

The Abelian final value Theorem (FVT) is the dual of the IVT $[38,43,46]$.

Theorem 4 (The final-value Theorem). Under the same assumptions of the IVT, we can state

$$
\lim _{t \rightarrow \infty} \frac{f(t)}{t^{\beta}}=\lim _{\sigma \rightarrow 0^{+}} \frac{\sigma^{\beta+1} F(\sigma)}{\Gamma(\beta+1)} .
$$

For proof, see [46] (section 8.7, pages 249-251). This result is useful in studying transient responses of systems. In particular, notice that $\lim _{t \rightarrow \infty} f(t)=\lim _{\sigma \rightarrow 0^{+}} \sigma F(\sigma)$ that expresses the classical FVT.

In table 1 , some examples of transforms are presented.

Table 1. Table with BLT pairs.

\begin{tabular}{ccc}
\hline$g(t)$ & $\mathcal{L} g(t)=G(s)$ & ROC \\
\hline$\delta(t)$ & 1 & $\mathbb{C}$ \\
\hline$\delta^{(n)}(t)$ & $s^{n}$ & $\mathbb{C}$ \\
\hline$\varepsilon(t)$ & $\frac{1}{s}$ & $\operatorname{Re}(s)>0$ \\
\hline$\varepsilon(-t)$ & $-\frac{1}{s}$ & $\operatorname{Re}(s)<0$ \\
\hline$t \varepsilon(t)$ & $\frac{1}{s^{2}}$ & $\operatorname{Re}(s)>0$ \\
\hline$\frac{1}{N !} e^{a t} \varepsilon(t)$ & $\frac{1}{(s-a)^{N+1}}$ & $\operatorname{Re}(s)>\operatorname{Re}(a)$ \\
\hline$-\frac{t^{N}}{N !} e^{a t} \varepsilon(-t)$ & $\frac{1}{(s-a)^{N+1}}$ & $\operatorname{Re}(s)<\operatorname{Re}(a)$ \\
\hline$\frac{t^{a}}{\Gamma(a+1)} \varepsilon(t)$ & $\frac{1}{s^{a+1}}$ & $\operatorname{Re}(s)>0$ \\
\hline$-\frac{t^{a}}{\Gamma(a+1)} \varepsilon(-t)$ & $\frac{1}{s^{a+1}}$ & $\operatorname{Re}(s)<0$ \\
\hline
\end{tabular}


Table 1. Cont.

\begin{tabular}{ccc}
\hline$e^{-|t|}$ & $\frac{2}{1-s^{2}}$ & $|\operatorname{Re}(s)|<1$ \\
\hline $\operatorname{sgn}(t) e^{-|t|}$ & $-\frac{s}{1-s^{2}}$ & $|\operatorname{Re}(s)|<1$ \\
\hline$e^{a t} \varepsilon(t)$ & $\frac{1}{s-a}$ & $\operatorname{Re}(s)>\operatorname{Re}(a)$ \\
\hline$-e^{a t} \varepsilon(-t)$ & $\frac{1}{s-a}$ & $\operatorname{Re}(s)<\operatorname{Re}(a)$ \\
\hline$\varepsilon(t)-\varepsilon(t-T)$ & $\frac{1-e^{-s T}}{s}$ & $\mathbb{C}$ \\
\hline $\cos \left(\omega_{0} t\right) \varepsilon(t)$ & $\frac{s}{s^{2}+\omega_{0}^{2}}$ & $\operatorname{Re}(s)>0$ \\
\hline $\sin \left(\omega_{0} t\right) \varepsilon(t)$ & $\frac{\omega_{0}}{s^{2}+\omega_{0}^{2}}$ & $\operatorname{Re}(s)>0$ \\
\hline$e^{-a t} \cos \left(\omega_{0} t\right) \varepsilon(t)$ & $\frac{(s+a)}{(s+a)^{2}+\omega_{0}^{2}}$ & $\operatorname{Re}(s)>-\operatorname{Re}(a)$ \\
\hline$e^{-a t} \sin \left(\omega_{0} t\right) \varepsilon(t)$ & $\frac{\omega_{0}}{(s+a)^{2}+\omega_{0}^{2}}$ & $\operatorname{Re}(s)>-\operatorname{Re}(a)$ \\
\hline
\end{tabular}

\subsection{Constructing the ULT}

As referred previously in Section 2.4, the ULT is a particular case of the BLT valid for functions identically null on $\mathbb{R}^{-}$(frequently using an imprecise term called as "causal functions"). The ULT is defined by $[37,38,47-50]$

$$
F_{u}(s)=\mathcal{L}_{u} f(t)=\int_{0}^{\infty} f(\tau) e^{-s \tau} \mathrm{d} \tau .
$$

However, its inverse is also given by the Bromwich integral

$$
f(t)=\frac{1}{2 \pi i} \int_{\gamma} F_{u}(s) e^{s t} \varepsilon(t) \mathrm{d} s,
$$

where the Heaviside unit step, $\varepsilon(t)$, is used to highlight that $f(t)$ is defined on $\mathbb{R}_{0}^{+}$.

Some properties of the ULT are slightly different from those of the BLT and can be read as:

1. Time derivative

This property is rather different from the corresponding of BLT. Let $N$ be a positive integer number. We have

$$
\mathcal{L}_{u} D^{N} f(t)=s^{N} F(s)-\sum_{k=0}^{N-1} s^{N-1-k} f^{(k)}\left(0^{+}\right) .
$$

This result has been used to solve initial value problems. In the integral case, we obtain

$$
\mathcal{L} D^{-N} f(t)=s^{-N} F(s) .
$$

2. Scale change

This property is only valid for $a>0$,

$$
\mathcal{L}[f(a t)]=\frac{1}{a} F(s / a) .
$$

3. Time shift

As the previous property, this is only valid fo $a>0$. If $a<0$, the property in not valid, which is inconvenient in applications.

$$
\mathcal{L}[f(t-a)]=e^{-a s} F(s) .
$$




\subsection{The Initial Condition Problem}

\subsubsection{Solution with the ULT}

The derivative property of the ULT is considered an important issue when solving initial value problems. In fact, since the works of Doetsch [37,38], the ULT has been used to solve linear differential equations under a given set of initial conditions (ICs), due to the derivative property (24).

Let us consider a causal stable linear system with transfer function $H(s)$ and assume that the input is an anti-causal function, $g(t)=0$ for $t>0$. The output, $f(t)$, is the inverse LT of $H(s) G(s)$. The initial value problem consists in computing $f(t)$ for $t>0$ from the values of $g(t)$ and $f(t)$ and their derivatives at $t=0$ (i.e., the free response). If there is no other input, then we can readily verify that $f\left(0^{-}\right)=f\left(0^{+}\right)$, but in general we have $g\left(0^{-}\right) \neq g\left(0^{+}\right)=0$. In case of existing another input for $t \geq 0$, (i.e., the forced response) we must compute the outputs separately.

Let us analyze the free response case. Traditionally, the problem has been solved with the help of (24). However, this relation was obtained under a classic approach and leads to strange results if we try to use it with distributions.

Example 2. Compute the ULT of the derivative of the Heaviside unit step. From (24), we have $\mathcal{L}_{u}[D \varepsilon(t)]=$ $s \frac{1}{s}-1=0$, which is obviously an unacceptable result.

Example 3. Let $f(t)=\frac{t^{\alpha-1}}{\Gamma(\alpha)} \varepsilon(t), \frac{1}{2}<\alpha<1$, with $L T$, for both transforms, given by $F(s)=\frac{1}{s^{\alpha}}, \operatorname{Re}(s)>0$. Let $0<\beta<\frac{1}{2}$. What is the LT of $g(t)=D^{\beta} f(t)$ ? By using the BLT we deduce immediately that $G(s)=$ $\frac{s^{\beta}}{s^{\alpha}}=s^{\alpha-\beta}$, from which we conclude that $g(t)=\frac{t^{\alpha-\beta-1}}{\Gamma(\alpha-\beta)} \varepsilon(t)$. The result is not so obvious when we use the ULT, because $f\left(0^{+}\right)=\infty$.

Besides this problem, (24) uses expressly the values of the function taken at $t=0^{+}$, which is not the correct option in practical applications. These difficulties led some researchers to propose a redefinition of the transform as [4,39]

$$
F_{u}(s)=\mathcal{L}_{u} f(t)=\int_{0^{-}}^{\infty} f(\tau) e^{-s \tau} \mathrm{d} \tau
$$

that leads to

$$
\mathcal{L} D^{N} f(t)=s^{N} F_{u}(s)-\sum_{k=0}^{N-1} s^{N-1-k} f^{(k)}\left(0^{-}\right)
$$

However, in the fractional case such formulation is incompatible with the Riemann-Liouville and Caputo derivatives that are defined only for $t=0^{+}$and does not generalize (29) for any real order. This means that the possible advantage of the ULT over the BLT disappears. On the other hand, (28) is incoherent with regard to the inversion transform. In fact, to compute the integral using the Cauchy residue theorem, we need to close the integration path: on the left, for $t>0$, and on the right, for $t<0$. This last case gives a null function, since the integrand is analytic and, consequently, its limit is always 0 as $t$ approaches $0^{-}$.

\subsubsection{Solution with the BLT}

For now, let us put aside the problem of $t=0^{+}$versus $t=0^{-}$and consider an application to give some additional insight for finding the most adequate formulation. Therefore, we have to avoid becoming tied to a formula that depends on a transform with limitations. Let us return to the general framework defined by the BLT where a function null for $t<0$ can be set as $f(t) \varepsilon(t)$. In general, this function is discontinuous at the origin, having a jump at $t=0$ with amplitude equal to $f(0)$ (the value $f(0)$, must be intended in distributional sense $[46,51,52]$ ). When we try to 
compute the derivative of this function, we need to remove such 'jump' to obtain a continuous function $f(t) \varepsilon(t)-f(0) \varepsilon(t)$ that has $f^{\prime}(t) \varepsilon(t)-f(0) \delta(t)$ for the first order derivative. The repetition of this procedure leads to so-called jump formula [52] that relates the derivative of a truncated function with the truncation of the $\mathrm{N}$-th order derivative:

$$
D^{N}[f(t) \cdot \varepsilon(t)]=\left[D^{N} f(t)\right] \cdot \varepsilon(t)+\sum_{i=0}^{N-1}\left[D^{N-1-k} f(0)\right] \cdot \delta^{(k)}(t), \quad N \in \mathbb{N} .
$$

Applying the BLT to this expression, we obtain

$$
\mathcal{L}\left[D^{N} f(t) \cdot \varepsilon(t)\right]=s^{N} \mathcal{L}[f(t) \cdot \varepsilon(t)]-\sum_{k=0}^{N-1}\left[D^{N-1-k} f(0)\right] s^{k},
$$

which is exactly the formula obtained with the unilateral LT, but without the constraint $t=0^{+}$vs. $t=0^{-}$. The jump formula was generalized to the fractional case in [40].

Theorem 5. Consider an increasing sequence of positive real numbers $\gamma_{k}, k=0,1, \cdots, N$. The fractional jump formula reads [40]

$$
f(t)^{\left(\gamma_{N}\right)} \cdot \varepsilon(t)=[f(t) \cdot \varepsilon(t)]^{\left(\gamma_{N}\right)}-\sum_{0}^{N-1} y^{\left(\gamma_{m}\right)}(0) \delta^{\left(\gamma_{N}-\gamma_{m}-1\right)}(t)
$$

We highlight the fact that the sequence $\gamma_{k}, k=0,1, \cdots, N$, is imposed by the particular application at hand, and not by the transform.

Theorem 6. Relation (32) leads to the generalization of the formula (31) so that

$$
\mathcal{L}\left[D^{\gamma_{N}} f(t) \cdot \varepsilon(t)\right]=s^{\gamma_{N}} \mathcal{L}[f(t) \cdot \varepsilon(t)]-\sum_{k=0}^{N-1} f^{\left(\gamma_{m}\right)}(0) s^{\gamma_{N}-\gamma_{m}-1}
$$

In the commensurate case we have $\gamma_{k}=k \alpha, k=0,1, \cdots, N$, and we obtain

$$
\mathcal{L}\left[D^{N \alpha} f(t) \cdot \varepsilon(t)\right]=s^{N \alpha} \mathcal{L}[f(t) \cdot \varepsilon(t)]-\sum_{k=0}^{N-1} f^{(k \alpha)}(0) s^{(N-k-1) \alpha} .
$$

Expression (34) must be compared with those we obtain using the ULT with the Riemann-Liouville and Caputo derivatives. We verify that they give rise to different formulae [40], namely

$$
\mathcal{L}\left[{ }^{R L} D^{\alpha} f(t)\right]=s^{\alpha} F(s)-\sum_{k=0}^{m-1} s^{k} D_{t}^{\alpha-k-1} f\left(0^{+}\right)
$$

and

$$
\mathcal{L}\left[{ }^{C} D^{\alpha} f(t)\right]=s^{\alpha} F(s)-\sum_{k=0}^{m-1} s^{\alpha-k-1} D^{k} f\left(0^{+}\right),
$$

where $m=\lceil\alpha\rceil$.

Example 4. To understand what is at stake, let us consider the linear system defined by the following differential equation:

$$
D^{2 \alpha} f(t)+a D^{\alpha} f(t)+b f(t)=g(t), a, b \in \mathbb{R},
$$


where $g(t)=0, t>0$ and $0<\alpha<1$. We want to compute the output $f(t)$ for $t>0$ using the ULT with the $R L$ or C derivatives and the BLT represented by (34)-(36), respectively. Therefore, they use the following IC

$$
\begin{aligned}
& U L T-R L: \quad f^{(\alpha-1)}\left(0^{+}\right) \quad \text { if } \alpha \leq \frac{1}{2} \\
& \text { ULT - RL : } f^{(\alpha-1)}\left(0^{+}\right), f^{(2 \alpha-1)}\left(0^{+}\right), f^{(2 \alpha-2)}\left(0^{+}\right) \text {if } \frac{1}{2}<\alpha \leq 1 \\
& \text { ULT }-C: \quad f\left(0^{+}\right) \quad \text { if } \alpha \leq \frac{1}{2} \\
& \text { ULT }-C: \quad f\left(0^{+}\right), f^{\prime}\left(0^{+}\right) \quad \text { if } \frac{1}{2}<\alpha \leq 1 \\
& \text { BLT : } \quad f(0), f^{(\alpha)}(0) \quad \text { if } 0<\alpha \leq 1 \text {. }
\end{aligned}
$$

It is important to note that with the BLT, we do not have to distinguish between the cases $\alpha \leq \frac{1}{2}$ and $\frac{1}{2}<\alpha \leq 1$.

For another comparison, let us introduce two state variables $v_{1}(t)=f(t)$ and $v_{2}(t)=D^{\alpha} f(t)$ so that the Equation (37) can be rewritten as

$$
\left[\begin{array}{l}
D^{\alpha} v_{1}(t) \\
D^{\alpha} v_{2}(t)
\end{array}\right]=\left[\begin{array}{rr}
0 & 1 \\
-b & -a
\end{array}\right]\left[\begin{array}{l}
v_{1}(t) \\
v_{2}(t)
\end{array}\right], \quad t>0
$$

Let $\mathbf{v}=\left[\begin{array}{l}v_{1}(t) \\ v_{2}(t)\end{array}\right]$. To solve the state Equation (39), using (34)-(36), we need the following IC

$$
\begin{array}{lll}
\text { ULT }-R L: & \mathbf{v}^{(\alpha-1)}\left(0^{+}\right) \\
\text {ULT }-C: & \mathbf{v}\left(0^{+}\right) & 0<\alpha \leq 1, \\
B L T: & \mathbf{v}(0) &
\end{array}
$$

respectively, or, attending to the definition of the vector $\mathbf{v}$, we need the IC

$$
\begin{array}{lll}
\text { ULT }-R L: & f^{(\alpha-1)}\left(0^{+}\right), f^{(2 \alpha-1)}\left(0^{+}\right) & \text {if } 0<\alpha \leq 1 \\
\text { ULT }-C: & f\left(0^{+}\right), f^{(\alpha)}\left(0^{+}\right) & \text {if } 0<\alpha \leq 1 \\
\text { BLT }: & f(0), f^{(\alpha)}(0) & \text { if } 0<\alpha \leq 1 .
\end{array}
$$

As observed, there is a contradiction between (38) and (40) in what regards the use of ULT with the RL and $C$ derivatives, while we have a perfect coherence when adopting the BLT.

\section{The Two-Dimensional LT}

\subsection{Definition}

We now introduce the two-dimensional BLT (2D-BLT). Let the function $e(x, t)=e^{s x+u t},(x, t) \in$ $\mathbb{R}^{2},(u, s) \in \mathbb{C}^{2}$, be the $2 \mathrm{D}$ exponential.

Definition 3. Similarly to the $1 D$ case, we define the 2D inverse BLT by [36,42-44]

$$
h(x, t)=\mathcal{L}_{s v}^{-1} \bar{H}(u, s)=\frac{1}{(2 \pi i)^{2}} \int_{\sigma_{1}-i \infty}^{\sigma_{1}+i \infty} \int_{\sigma_{2}-i \infty}^{\sigma_{2}+i \infty} \bar{H}(u, s) e^{u x+s t} \mathrm{~d} u \mathrm{~d} s,(x, t) \in \mathbb{R}^{2},
$$

where $\bar{H}(u, s)$ is the $2 D-B L T$ of $h(x, t)$. Moreover, the integration paths are vertical straight lines with abscissas $\sigma_{1}$ and $\sigma_{2}$, that are located inside a region where $\bar{H}(u, s)$ is analytic. For simplicity, we may denote those straight lines by $\gamma_{1}$ and $\gamma_{2}$. The Cauchy principal values of the integrals are assumed in (41) and in the following.

In coherence with previous sections, the partial transform relatively to only one of the variables will be represented as $\mathcal{L}_{x} h(x, t)=H(u, t)$ or $\mathcal{L}_{t} h(x, t)=H(x, s)$. 
Definition 4. The 1D-BLT studied above suggests us to introduce the 2D-BLT by

$$
\mathcal{L}_{x t}[h(x, t)]=\bar{H}(u, s)=\int_{-\infty}^{\infty} \int_{-\infty}^{\infty} h(x, t) e^{-u x-s t} \mathrm{~d} x \mathrm{~d} t, u, s \in \mathbb{C} .
$$

Consider the partial transforms $\mathcal{L}_{x} h(x, t)=H(s, t)$ and $\mathcal{L}_{t} h(x, t)=H(x, v)$. We define the sequential transforms (also called iterated transforms [53], but we reserve this name for another transform (67)), by

$$
\begin{aligned}
& \mathcal{L}_{t} \mathcal{L}_{x}\{f\}=\int_{-\infty}^{\infty} e^{-s t} d t \int_{-\infty}^{\infty} e^{-s x} f(x, t) d x, \\
& \mathcal{L}_{x} \mathcal{L}_{t}\{f\}=\int_{-\infty}^{\infty} e^{-u x} d x \int_{-\infty}^{\infty} e^{-s t} f(x, t) d t .
\end{aligned}
$$

It may eventually happen that the 2D-BLT exists and is equal to one or two iterated transforms, but not necessarily. However, if the 2D-BLT converges absolutely, then the iterated transform exists and the three transforms are identical [53]. In most practical situations, we have to use two iterated transforms, for ease of calculation and introduction of the IC.

Remark 4. The separable case $h(x, t)=f(x) g(t)$ is straightforward giving

$$
\mathcal{L}_{x y}[f(x) g(t)]=F(s) G(v) .
$$

Example 5. If $h(x, t)=e^{-2|x|} \mathcal{E}(t)$, then we have

$$
\begin{gathered}
H(x, s)=\mathcal{L}_{t} h(x, t)=e^{-2|x|} \frac{1}{s}, \operatorname{Re}(s)>0, \\
\bar{H}(u, s)=\frac{1}{s\left(u^{2}-4\right)},|\operatorname{Re}(u)|<2 \text { and } \operatorname{Re}(s)>0 .
\end{gathered}
$$

Attending to the existence conditions of the 1D-BLT we can state sufficient conditions for the existence of the 2D-BLT.

Theorem 7. Let $h(x, t)$ be a function piecewise continuous, with bounded variation, locally integrable (in the sense that the function is absolutely integrable in any rectangle, $[a, b] \times[c, d]$, so that $\left.\int_{a}^{b} \int_{c}^{d}|h(x, t)| \mathrm{d} x \mathrm{~d} t<\infty\right)$ and of exponential order, that is to say, that it does not "grow faster" than given exponentials, then the 2D-LT exists and is analytic in vertical strips in the complex plane defined by $b_{1}<\operatorname{Re}(s)<a_{1}$ and $b_{2}<\operatorname{Re}(v)<a_{2}$. These strips define regions where the ROC are located.

This means that:

1. There are real constants $A$ and $a_{1}, a_{2}>0$ such that $|h(x, t)|<A \cdot e^{a_{1} x+a_{2} t}$, when $x$ and $t$ are large and negative (say, for $x<x_{1}, t<t_{1} \in \mathbb{R}$ ).

2. There are real constants $B$ and $b_{1}, b_{2}>0$ such that $|h(x, t)|<B \cdot e^{b_{1} x+b_{2} t}, x$ and $t$ are large (say, for $\left.x>x_{1}, t>t_{1} \in \mathbb{R}\right)$.

3. It also has to be true that $a_{1}>b_{1}$ and $a_{2}>b_{2}$.

The proof of the Theorem arises immediately from the hypotheses.

\subsection{Properties of the $2 D-B L T$}

Most of the properties of the 2D-LT are a direct adaptation of similar ones from the 1D-LT. Consequently, the properties for the 2D-LT are: 
1. Linearity/homogeneity

Let a function $\mathcal{L} g(x, t)=G(u, s)$. We have

$$
\mathcal{L}[a f(x, t)+b g(x, t)]=a F(u, s)+b G(u, s) .
$$

2. Fractional order derivatives

As we are working with derivatives defined on $\mathbb{R}$, we can write $D_{x}^{\alpha} e^{u x+s t}=u^{\alpha} e^{u x+s t}$ for $\operatorname{Re}(u)>0$ or $\operatorname{Re}(u)<0$ depending on the causality of the derivative [45]. Similarly, we have $D_{t}^{\beta} e^{u x+s t}=$ $s^{\beta} e^{u x+s t}$ for $\operatorname{Re}(s)>0$ or $\operatorname{Re}(s)<0$. We can write

$$
D_{x}^{\alpha} D_{y}^{\beta}[h(x, t)]=\frac{1}{(2 \pi i)^{2}} \int_{\gamma_{1}} \int_{\gamma_{2}} s^{\alpha} v^{\beta} \bar{H}(u, s) e^{u x+s t} \mathrm{~d} s \mathrm{~d} v,
$$

from where we deduce that,

$$
\mathcal{L}_{x t}\left[D_{x}^{\alpha} D_{t}^{\beta} h(x, t)\right]=s^{\alpha} v^{\beta} \bar{H}(u, s) .
$$

This property is valid for any $\alpha$ and $\beta$, either real or complex.

Example 6. Let us consider the 2D Heaviside unit step

$$
\varepsilon(x, t)= \begin{cases}1 & x \geq 0, \text { and } t \geq 0 \\ 0 & x<0, \text { or } t<0\end{cases}
$$

and the power function

$$
h(x, t)=\left[\frac{x^{\alpha}}{\Gamma(\alpha+1)} \frac{t^{\beta}}{\Gamma(\beta+1)}\right] \varepsilon(x, t), \alpha>-1, \beta>-1 .
$$

The $2 D-L T$ of $h(x, t)$ is given by

$$
\mathcal{L}_{x t}\left[\frac{x^{\alpha}}{\Gamma(\alpha+1)} \frac{t^{\beta}}{\Gamma(\beta+1)} \varepsilon(x, t)\right]=\frac{1}{s^{\alpha+1} v^{\beta+1}}, \quad \operatorname{Re}(u)>0, \operatorname{Re}(s)>0 .
$$

Definition 5. This property suggests us to introduce a 2D Mittag-Leffler function [54-58] by

$$
E_{\alpha \beta}(x, t)=\sum_{n=0}^{\infty} \frac{x^{n \alpha} t^{n \beta}}{\Gamma\left(\alpha n+\alpha_{0}\right) \Gamma\left(\beta n+\beta_{0}\right)} \varepsilon(x, t), \quad 0<\alpha_{0}, \beta_{0} \leq 1,
$$

which is slightly different than the 4-parameter Mittag-Leffler function formulated in [57]. If $\alpha_{0}, \beta_{0}=1$ than the $2 D-B L T$ of $E_{\alpha \beta}(x, t)$ in (50) is given by:

$$
\mathcal{L}_{x t}\left[E_{\alpha \beta}(x, t)\right]=\frac{u^{\alpha-1} s^{\beta-1}}{u^{\alpha} s^{\beta}-1}, \operatorname{Re}(u)>0, \operatorname{Re}(s)>0 .
$$

3. Rotation rule

This rule is a generalization of the scale change property [36,53]. In this case, we can compute the 2D-LT of the function:

$$
g(x, t)=h\left(a_{1} x+b_{1} y, a_{2} x+b_{2} y\right) .
$$

We have

$$
\bar{G}(u, s)=\int_{R} \int_{R} h\left(a_{1} x+b_{1} y, a_{2} x+b_{2} y\right) e^{-s x-v y} \mathrm{~d} x \mathrm{~d} t .
$$


Let us introduce $d_{a b}=a_{1} b_{2}-a_{2} b_{1}$, and the pairs $\left(x^{\prime}, t^{\prime}\right)=\left(a_{1} x+b_{1} y, a_{2} x+b_{2} y\right)$ and $\left(u^{\prime}, s^{\prime}\right)=$ $\left(\frac{b_{2} u-a_{2} s}{d_{a b}}, \frac{-b_{1} u+a_{1} s}{d_{a b}}\right)$, such that

$$
u x+s t=u^{\prime} x^{\prime}+s^{\prime} t^{\prime} .
$$

As the Jacobian of the transformation is equal to $\frac{1}{d_{a b}}$, we obtain

$$
\bar{G}(u, s)=\left|\frac{1}{d_{a b}}\right| \bar{H}\left(u^{\prime}, s^{\prime}\right) .
$$

The dual property can be obtained using a similar procedure in the inverse transform.

Remark 5. Letting $b_{1}=a_{2}=0$, we obtain the scale change property.

4. Shift rules

This is similar to the analogue property of 1D-BLT and is readily obtained from the transform or inverse definitions. If $(a, b) \in \mathbb{R}^{2}$, then

$$
\mathcal{L}[f(x-a, t-b)]=e^{-a u-b s} \bar{F}(u, s) .
$$

The dual property is called modulation property and reads:

$$
\mathcal{L}_{x t}\left[e^{a x+b t} f(x, t)\right]=\bar{F}(u-a, s-b) .
$$

This property remains valid when $a$ and $b$ are complex numbers.

Example 7. Consider the function defined in (49). With the dual of (52) we obtain

$$
\mathcal{L}_{x t}\left[\frac{x^{\alpha} t^{\beta} e^{a x+b t}}{\Gamma(\alpha+1) \Gamma(\beta+1)} \varepsilon(x, t)\right]=\frac{1}{(u-a)^{\alpha+1}(s-b)^{\beta+1}},
$$

for $\operatorname{Re}(u)>\operatorname{Re}(a)$ and $\operatorname{Re}(s)>\operatorname{Re}(b)$.

Remark 6. We can combine shifts with rotation to obtain a more general transformation [53].

5. Convolution

Let us define, with all generality, the convolution between two functions $f(x, t)$ and $g(x, t)$ by:

$$
f(x, t) * * g(x, t)=\int_{-\infty}^{\infty} \int_{-\infty}^{\infty} f(\xi, \zeta) g(x-\xi, t-\zeta) \mathrm{d} \xi \mathrm{d} \zeta,
$$

where the symbol $* *$ stands for two-dimensional convolution, and insert expression (52) so that we can write

$$
\begin{gathered}
f(x, t) * * g(x, t)= \\
\int_{-\infty}^{\infty} \int_{-\infty}^{\infty} f(\xi, \zeta) \frac{1}{(2 \pi i)^{2}} \int_{\gamma_{1}} \int_{\gamma_{2}} \bar{G}(u, s) e^{u x+s t} e^{-u \tilde{\zeta}-s \zeta} \mathrm{d} u \mathrm{~d} s \mathrm{~d} \xi \mathrm{d} \zeta .
\end{gathered}
$$

Interchanging the order of integration, we get:

$f(x, t) * * g(x, t)=$

$$
\frac{1}{(2 \pi i)^{2}} \int_{\gamma_{1}} \int_{\gamma_{2}} \bar{G}(u, s) e^{u x+s t} \int_{-\infty}^{\infty} \int_{-\infty}^{\infty} f(\xi, \zeta) e^{-u \xi-s \zeta} \mathrm{d} \xi \mathrm{d} \zeta \mathrm{d} u \mathrm{~d} s
$$


that leads to

$$
\mathcal{L}_{x t}[f(x, t) * * g(x, t)]=\bar{F}(u, s) \bar{G}(u, s) .
$$

We can obtain the dual property, that is interesting in 2D-FT. Nonetheless, we shall not explore this further in the scope of this paper, but the topic is of interest for a specific study [59].

Example 8. Let $\alpha>0, \beta>0$ and

$$
\bar{F}(u, s)=\frac{\bar{G}(u, s)}{u^{\alpha} s^{\beta}}, \operatorname{Re}(u)>0, \operatorname{Re}(s)>0 .
$$

Then the inverse $2 D-L T$ of $\bar{F}(u, s)$ is given by [53]

$$
f(x, t)=\frac{1}{\Gamma(\alpha) \Gamma(\beta)} \int_{-\infty}^{x} \int_{-\infty}^{t} g(v, w)(x-v)^{\alpha-1}(t-w)^{\beta-1} \mathrm{~d} v \mathrm{~d} w,
$$

that may be considered as a $2 D$ Liouville anti-derivative of order $(\alpha, \beta)[6,45,60]$.

Example 9. This property can be extended to a different convolution definition, called convolution about an axis, given by [53]

$$
f(x, t) *_{a b} g(x, t)=\int_{-\infty}^{\infty} f(\xi) g(x-a \xi, t-b \xi) \mathrm{d} \xi .
$$

We have

$$
\mathcal{L}_{x t}\left[\int_{-\infty}^{\infty} f(\xi) g(x-a \xi, t-b \xi) \mathrm{d} \xi\right]=F(a s+b v) \bar{G}(u, s) .
$$

If $f(x)=\frac{x^{\alpha}}{\Gamma(\alpha+1)} \varepsilon(x)$, then we obtain

$$
\mathcal{L}_{x t}\left[\int_{0}^{\infty} \frac{\xi^{\alpha}}{\Gamma(\alpha+1)} g(x-a \xi, t-b \xi) \mathrm{d} \xi\right]=\frac{\bar{G}(u, s)}{(a u+b s)^{\alpha+1}} .
$$

\subsection{Special Cases}

We now present some cases of particular interest:

1. The 2D-FT and the mixed 2D-LFT

With the formulation that we have proposed, the Fourier transforms are particular cases of the Laplace transforms. The 2D-FT can be written as

$$
\bar{H}(i v, i \omega)=\int_{-\infty}^{\infty} \int_{-\infty}^{\infty} h(x, t) e^{-i v x-i \omega t} \mathrm{~d} x \mathrm{~d} t, \quad v, \omega \in \mathbb{R} .
$$

In some applications, as the diffusion, it is convenient to use a mixed LT-FT transform that we can state as:

$$
\bar{H}(i v, s)=\int_{-\infty}^{\infty} \int_{-\infty}^{\infty} h(x, t) e^{-i v x-s t} \mathrm{~d} x \mathrm{~d} t, v \in \mathbb{R}, s \in \mathbb{C} .
$$


2. One-sided 2D-LT

If $f(x, t)$ is non null only in the first quadrant, then we obtain the 2D-ULT

$$
\mathcal{L}_{x t}[h(x, t) \varepsilon(x, t)]=\bar{H}_{u}(u, s)=\int_{0}^{\infty} \int_{0}^{\infty} h(x, t) e^{-u x-s t} \mathrm{~d} x \mathrm{~d} t, u, s \in \mathbb{C} .
$$

This expression is the one used traditionally for the 2D-LT.

3. Function defined on a straight line

Consider the function $f(x, t)=h(x \mp t)$. Its 2D-BLT is given by

$$
\bar{F}(u, s)=H(u) \int_{\mathbb{R}} e^{-(u \pm s) t} \mathrm{~d} t .
$$

The integral is divergent. However, if $\operatorname{Re}(s)=\mp \operatorname{Re}(u)$, with $\operatorname{Im}(u)=v$ and $\operatorname{Im}(s)=\omega$, and knowing the properties of the Dirac distribution, then we get

$$
\bar{F}(u, s)=H(u) \delta(v \pm \omega) .
$$

Therefore, we can consider that the 2D-LT of $h(x-t)$ is a continuum of impulses.

This example seems to be more interesting when considered from the 2D-FT. In this case, we have

$$
\bar{H}(i v, i \omega)=H(i v) \delta(v \pm \omega) .
$$

This example deserves some attention when the function is defined in the first quadrant: $f(x, t)=$ $h(x-t) \varepsilon(x, t)$. We have

$$
\bar{F}(u, s)=\int_{0}^{\infty} \int_{0}^{\infty} h(x-t) e^{-u x-s t} \mathrm{~d} x \mathrm{~d} t=\int_{0}^{\infty} \int_{-t}^{\infty} h(x) e^{-u x} \mathrm{~d} x e^{-(u+s) t} \mathrm{~d} t .
$$

Let $H_{u}(s)$ be the ULT of $h(x)$. We can write

$$
\bar{F}(u, s)=\int_{0}^{\infty}\left[H_{u}(s)+\int_{-t}^{0} h(x) e^{-u x} \mathrm{~d} x\right] e^{-(u+s) t} \mathrm{~d} t=\frac{H_{u}(s)}{u+s}+\int_{0}^{\infty} \int_{-t}^{0} h(x) e^{-u x} \mathrm{~d} x e^{-(u+s) t} \mathrm{~d} t .
$$

We need to compute the second parcel separately and we obtain:

$$
\int_{0}^{\infty} \int_{-t}^{0} h(x) e^{-u x} \mathrm{~d} x e^{-(u+s) t} \mathrm{~d} t=\int_{0}^{\infty} h(-x) \int_{-t}^{0} \varepsilon(t-x) e^{-(u+s) t} \mathrm{~d} t e^{-u x} \mathrm{~d} x=\int_{0}^{\infty} h(-x) \frac{e^{-s x}}{u+s} \mathrm{~d} x .
$$

There are two particular cases that simplify the result, leading to

$$
\bar{F}(u, s)= \begin{cases}\frac{H_{u}(u)+H_{u}(s)}{u+s}, & h \text { is even } \\ \frac{H_{u}(u)-H_{u}(s)}{u+s}, & h \text { is odd } .\end{cases}
$$

Example 10. Let $f(x, t)=\varepsilon(x, t)$. We have successively

$$
\begin{aligned}
& \bar{F}(u, s)=\int_{0}^{+\infty} e^{-u x} d x \int_{0}^{+\infty} e^{-s t} \varepsilon(x-t) d t=\int_{0}^{+\infty} e^{-u x} d x \int_{0}^{x} e^{-s t} d t \\
& \bar{F}(u, s)=\int_{0}^{+\infty} e^{-u x}\left(1-e^{-(u+s) x}\right) d x=\frac{1}{u(u+s)} \operatorname{Re}(u), \operatorname{Re}(s)>0 .
\end{aligned}
$$


4. The $2 \mathrm{D}$ to $1 \mathrm{D}$ reduction

Consider $h_{2}(x, t)$ and define a single variable function $h_{1}(t)$ given by

$$
h_{1}(t)=h_{2}(t, t),
$$

and having BLT, $H_{1}(s)$, called associated transform of $H_{2}(u, s)$. The process of going from $H_{2}$ to $H_{1}$ is the $2 D$-to-1D reduction. This procedure is used in the study of linear time varying systems [61]. As $h_{1}(t)=h_{2}(t, t)$, we can use the inverse LT integrals to write

$$
\int_{\gamma_{1}} H_{1}(s) e^{s t} \mathrm{~d} s=\frac{1}{2 \pi i} \int_{\gamma_{2}} \int_{\gamma_{3}} \bar{H}_{2}(u, v) e^{(u+v) t} \mathrm{~d} u \mathrm{~d} v, t \in \mathbb{R} .
$$

Computing the 1D-BLT of both sides of this equation, we obtain

$$
H_{1}(s)=\int_{-\infty}^{\infty} \frac{1}{2 \pi i} \int_{\gamma_{2}} \int_{\gamma_{3}} \bar{H}_{2}(u, v) e^{(u+v) t} \mathrm{~d} u \mathrm{~d} v e^{-s t} \mathrm{~d} t, s \in \mathbb{C} .
$$

Assume that all involved ROC include the imaginary axis. In such case, we can set $\gamma_{1}=\gamma_{2}=$ $\gamma_{3}=0$ and put $s=i \omega, u=i v$ and $v=i \mu$. Substituting and interchanging the sequence of integration, we can write

$$
H_{1}(i \omega)=\frac{1}{2 \pi} \int_{-\infty}^{\infty} \int_{-\infty}^{\infty} \bar{H}_{2}(i v, i \mu) \int_{-\infty}^{\infty} e^{-i(\omega-v-\mu) t} \mathrm{~d} t \mathrm{~d} u \mathrm{~d} v, \omega \in \mathbb{R} .
$$

As $\int_{-\infty}^{\infty} e^{-i(\omega-v-\mu) t} \mathrm{~d} t=\delta(\omega-v-\mu)$, we obtain

$$
H_{1}(i \omega)=\frac{1}{2 \pi} \int_{-\infty}^{\infty} \int_{-\infty}^{\infty} \bar{H}_{2}(i v, i \mu) \delta(\omega-v-\mu) \mathrm{d} v \mathrm{~d} \mu,
$$

and finally

$$
H_{1}(i \omega)=\frac{1}{2 \pi} \int_{-\infty}^{\infty} \bar{H}_{2}(i v, i \omega-i v) \mathrm{d} v .
$$

The dual of this property can be obtained in a similar way.

5. The iterated LT

The iterated LT is the dual of the previous property.

Definition 6. The iterated LT is a particular case of the 2D-BLT (42) obtained with $u=s$, leading to [61]

$$
\mathcal{L}_{I T}[h(x, t)]=\bar{H}(s)=\int_{-\infty}^{\infty} \int_{-\infty}^{\infty} h(x, t) e^{-s(x+t)} \mathrm{d} x \mathrm{~d} t, s \in \mathbb{C} .
$$

We can obtain $h(t)=\mathcal{L}_{t}^{-1} \bar{H}(s)$ by a procedure similar to the one used for deducing (66).

\section{The 2D Fractional Linear Systems}

\subsection{On the 2D Fractional Derivatives}

In (58) we introduced the 2D anti-derivative. After some changes of variables we can redefine it by [60]

$$
D_{x t}^{\alpha, \beta} f(x, t)=\frac{1}{\Gamma(-\alpha) \Gamma(-\beta)} \int_{0}^{\infty} \int_{0}^{\infty} f(x-v, t-w) v^{-\alpha-1} w^{-\beta-1} \mathrm{~d} v \mathrm{~d} w
$$


where $\alpha, \beta<0$. For $\alpha, \beta>0$, the integrals are singular and need to be regularized $[62,63]$. If $0<\alpha, \beta \leq 1$, then we can write

$$
D_{x t}^{\alpha, \beta} f(x, t)=\frac{1}{\Gamma(-\alpha) \Gamma(-\beta)} \int_{0}^{\infty} \int_{0}^{\infty}[f(x-v, t-w)-f(x, t)] v^{-\alpha-1} w^{-\beta-1} \mathrm{~d} v \mathrm{~d} w .
$$

However, the Grünwald-Letnikov formulation (2DGL) may be more interesting. It reads [60]:

$$
D_{x t}^{\alpha, \beta} f(x, t)=\lim _{\left(h_{1}, h_{2}\right) \rightarrow\left(0^{+}, 0^{+}\right)} h_{1}^{\alpha} h_{2}^{\beta} \sum_{n=0}^{\infty} \sum_{m=0}^{\infty} \frac{(-\alpha)_{n}}{n !} \frac{(-\beta)_{m}}{m !} f\left(x-n h_{1}, t-m h_{2}\right) .
$$

The transfer function of these derivatives is given by the 2D-BLT of (68) and (69). To obtain them, we set $f(x, t)=e^{u x+s t}$ in the above expressions. For example, for the 2D-GL, we have successively:

$$
\begin{gathered}
\mathcal{L}_{x t}\left[D_{x t}^{\alpha, \beta} e^{u x+s t}\right]=e^{u x+s t} \lim _{\left(h_{1}, h_{2}\right) \rightarrow\left(0^{+}, 0^{+}\right)} h_{1}^{-\alpha} h_{2}^{-\beta} \sum_{n=0}^{\infty} \sum_{m=0}^{\infty} \frac{(-\alpha)_{n}}{n !} \frac{(-\beta)_{m}}{m !} e^{-n u h_{1}-m s h_{2}}= \\
e^{u x+s t} \lim _{h_{1} \rightarrow 0^{+}} h_{1}^{\alpha} \sum_{n=0}^{\infty} \frac{(-\alpha)_{n}}{n !} e^{-n u h_{1}} \lim _{h_{2} \rightarrow 0^{+}} h_{2}^{\beta} \sum_{m=0}^{\infty} \frac{(-\beta)_{m}}{m !} e^{-m s h_{2}}= \\
e^{u x+s t} \lim _{h_{1} \rightarrow 0^{+}}\left[\frac{1-e^{-u h_{1}}}{h_{1}}\right]^{\alpha} \lim _{h_{2} \rightarrow 0^{+}}\left[\frac{1-e^{-s h_{2}}}{h_{2}}\right]^{\beta} .
\end{gathered}
$$

Therefore, the transfer function, $H(u, s)$, of the 2D derivative is

$$
H(u, s)=u^{\alpha} s^{\beta}, \operatorname{Re}(u), \operatorname{Re}(s)>0 .
$$

We can use the "right" derivative instead of the left that we adopted.

\subsection{System Definition}

Let us consider the Mittag-Leffer function [54-56,58] and the 2D Mittag-Leffer function introduced in (51). We can interpret it as the solution of the differential equation

$$
D_{x t}^{\alpha, \beta} h(x, t)+h(x, t)=D_{x t}^{\alpha, \beta} f(x, t)
$$

when the input is $f(x, t)=\varepsilon(x, t)$.

Definition 7. This suggests us to introduce the concept of fractional 2D linear system by

$$
\sum_{k=0}^{N} \sum_{j=0}^{N} a_{k, j} D_{x t}^{\alpha_{k}, \beta_{j}} f(x, t)=\sum_{k=0}^{M} \sum_{j=0}^{M} b_{k, j} D_{x t}^{\alpha_{k}, \beta_{j}} g(x, t),
$$

where $a_{k, j}$ and $b_{k, j}, k=0,1, \cdots$, are real equation coefficients, with $a_{N, N}=1$. Additionally, $N$ and $M$ are non negative integers verifying $M \leq N$. Note that there is no inconvenient in choosing equal summation limits, because we can set some coefficients or orders equal to zero. The derivative orders $\alpha_{k}, k=0,1, \cdots$ and $\beta_{k}, k=0,1, \cdots$, form two non decreasing sequences of positive real numbers.

The corresponding transfer function is the eigenvalue corresponding to the eigenfunction $e(x, t)=$ $e^{s x+u t},(x, t) \in \mathbb{R}^{2},(u, s) \in \mathbb{C}^{2}$. It is given by

$$
H(u, s)=\frac{\sum_{k=0}^{M} \sum_{j=0}^{M} b_{k, j} u^{\alpha_{k}} \beta^{\beta_{j}}}{\sum_{k=0}^{N} \sum_{j=0}^{N} a_{k, j} u^{\alpha_{k} \beta^{\beta_{j}}}} .
$$


In practical applications, the input function assumes frequently the form $g(x, t)=v(t) \delta(x)$, where $v(t)$ is a function with BLT.

The general formulation stated in (71) and (72) is dificult to treat. The more manageable commensurate case reads

$$
H(u, s)=\frac{\sum_{k=0}^{M} \sum_{j=0}^{M} b_{k, j} u^{k \alpha}{ }_{S}^{j \beta}}{\sum_{k=0}^{N} \sum_{j=0}^{N} a_{k, j} u^{k \alpha}{ }_{S} j \beta},
$$

where $0<\alpha, \beta \leq 1$. If $u=i v$ and $s=i \omega$, then we obtain the frequency response of the system that is given by:

$$
H(u, s)=\frac{\sum_{k=0}^{M} \sum_{j=0}^{M} b_{k, j}(i v)^{k \alpha}(i \omega)^{j \beta}}{\sum_{k=0}^{N} \sum_{j=0}^{N} a_{k, j}(i v)^{k \alpha}(i \omega)^{j \beta}}
$$

in the commensurate case. This frequency response is also called bi-spectrum.

\subsection{The Initial and Final Value Theorems}

When studying the BLT we introduced the initial and final value theorems. We now address their generalization to the $2 \mathrm{D}$ case. We start by considering the initial value and noting that we can apply (20) to each of the partial transforms (43) we obtain

$$
\begin{gathered}
f(x, t) \sim A(x) \varphi(t) \text { for } t \rightarrow 0 \Longrightarrow F(x, \sigma) \sim A(x) \Phi(\sigma) \text { for } \sigma \rightarrow \infty \\
f(x, t) \sim B(t) \psi(x) \text { for } t \rightarrow 0 \Longrightarrow F(u, t) \sim B(t) \Psi(v) \text { for } v \rightarrow \infty .
\end{gathered}
$$

Choosing $\psi(x)=x^{\alpha} \varepsilon(x)$ and $\varphi(t)=t^{\beta} \varepsilon(t)$, we can formulate the $2 \mathrm{D}$ initial-value theorem.

Theorem 8 (The 2D initial-value Theorem). Assume that $f(x, t)$ is a causal function such that in some neighborhood of the origin it is a regular distribution, corresponding to an integrable function in both $x$ and $t$, and that the $L T$ are $F(u, t)$ and $F(x, s)$ with $\operatorname{ROC} \operatorname{Re}(u)>0$ and $\operatorname{Re}(s)>0$.

In addition, assume that there are a real numbers $\alpha, \beta>-1$, such that $\lim _{x, t \rightarrow 0^{+}} \frac{f(x, t)}{x^{\alpha} t^{\beta}}$ exists and is a finite complex value. Then, we have

$$
\lim _{x, t \rightarrow 0^{+}} \frac{f(x, t)}{x^{\alpha} t^{\beta}}=\lim _{v, \sigma \rightarrow \infty} \frac{\sigma^{\alpha+1} \sigma^{\beta+1} \bar{F}(v, \sigma)}{\Gamma(\alpha+1) \Gamma(\beta+1)} .
$$

In a similar way, we can revert the limit computations obtaining the 2D final-value theorem:

Theorem 9 (The 2D final-value Theorem). Assume that $f(x, t)$ is a causal function such that it has the asymptotic property $f(x, t) \sim A x^{\alpha} t^{\beta}$ and the $L T$ are $F(u, t)$ and $F(x, s)$ with $\operatorname{ROC} \operatorname{Re}(u)>0$ and $\operatorname{Re}(s)>0$.

Then, we have

$$
\lim _{x, t \rightarrow \infty} \frac{f(x, t)}{x^{\alpha} t^{\beta}}=\lim _{v, \sigma \rightarrow 0^{+}} \frac{\sigma^{\alpha+1} \sigma^{\beta+1} \bar{F}(v, \sigma)}{\Gamma(\alpha+1) \Gamma(\beta+1)} .
$$

\subsection{Initial Conditions}

In the 2D LT, the IC problem is similar to the 1D LT studied in Section 2.5. The application to 2D systems is immediate, if they exist only for one variable. In the most common situations such variable is time. Therefore, we can adapt the formula (33) to obtain

$$
\mathcal{L}_{t}\left[D_{t}^{\gamma_{N}} f(x, t) \cdot \varepsilon(t)\right]=s^{\gamma_{N}} \mathcal{L}_{t}[f(x, t) \cdot \varepsilon(t)]-\sum_{k=0}^{N-1} f^{\left(\gamma_{m}\right)}(x, 0) s^{\gamma_{N}-\gamma_{m}-1}
$$


However, if the function at hand is non null only in the first quadrant (we could consider others), then the solution is not readily obtained from (77). To obtain the result we are looking for, we proceed heuristically. Let us assume that we want to solve a simple equation like

$$
D_{x t}^{\alpha, \beta} h(x, t)+a h(x, t)=f(x, t), a \in \mathbb{R},
$$

for $x>0, t>0$. Considering $f(x, t)=0$ on such domain we have:

$$
D_{x t}^{\alpha, \beta} h(x, t)+a h(x, t)=0, \text { for } x>0, t>0, a \in \mathbb{R} .
$$

This is equivalent to say that $h(x, t)$ is defined on $\mathbb{R}^{2}$, but it is only observed on $\mathbb{R}^{+} \times \mathbb{R}^{+}$through the "observation window" represented by the 2D Heaviside function:

$$
\left[D_{x t}^{\alpha, \beta} h(x, t)\right] \varepsilon(x, t)+a h(x, t) \varepsilon(x, t)=0 .
$$

To solve the problem, we need to relate $\left[D_{x t}^{\alpha, \beta} h(x, t)\right] \varepsilon(x, t)$ with $D_{x t}^{\alpha, \beta}[h(x, t) \varepsilon(x, t)]$. We start by noting that $h(x, t) \varepsilon(x, t)$ is discontinuous near the origin, which implies that it is convenient to remove the discontinuity before doing the derivative computation,. This means that we need to subtract the corresponding "jump" given by $h(0,0) \varepsilon(x, t)$. To clarify the question and motivated by (51) we assume that the solution of (78) has the form:

$$
h(x, t)=\sum_{n=0}^{\infty} A_{n} \frac{x^{n \alpha} t^{n \beta}}{\Gamma(\alpha n+1) \Gamma(\beta n+1)} \varepsilon(x, t),
$$

which can be transformed by the 2D-BLT into the relation:

$$
\bar{H}(u, s)=\sum_{n=0}^{\infty} A_{n} u^{-n \alpha-1} s^{-n \beta-1} .
$$

On the other hand, the 2D-BLT of (78) gives

$$
u^{\alpha} s^{\beta} \bar{H}(u, s)+a \bar{H}(u, s)=0 .
$$

Therefore, inserting (81) in (82), we obtain:

$$
\begin{gathered}
\sum_{n=0}^{\infty} A_{n} u^{-(n-1) \alpha-1} s^{-(n-1) \beta-1}+a \sum_{n=0}^{\infty} A_{n} u^{-n \alpha-1} s^{-n \beta-1}=0 . \\
A_{0} u^{\alpha-1} s^{\beta-1}+\sum_{n=0}^{\infty} A_{n+1} u^{-n \alpha-1} s^{-n \beta-1}+\sum_{n=0}^{\infty} a A_{n} u^{-n \alpha-1} s^{-n \beta-1}=0,
\end{gathered}
$$

from where we deduce that

$$
\begin{gathered}
A_{n+1}=-a A_{n}, n=0,1,2, \cdots \\
A_{n}=A_{0}(-a)^{n}, n=1,2, \cdots
\end{gathered}
$$

However, in (84) there is a non compensated term $A_{0} u^{\alpha-1} s^{\beta-1}$ that has to be removed from the Equation (82). Noting that from (80) we have $A_{0}=h(0,0)$, and the expression $A_{0} u^{\alpha-1} s^{\beta-1}$ is the 2D-BLT of $h(0,0) D_{x t}^{\alpha, \beta} \varepsilon(x, t)$, then the Equation (79) must be modified to include the IC:

$$
D_{x t}^{\alpha, \beta}[h(x, t) \varepsilon(x, t)]-h(0,0) \varepsilon(x, t)+a h(x, t) \varepsilon(x, t)=0, \text { for } x>0, t>0, a \in \mathbb{R} .
$$


Therefore, we conclude that a term of the form $\left[D_{x t}^{\alpha_{1}, \beta_{1}} h(x, t)\right] \varepsilon(x, t)$ must be substituted by $D_{x t}^{\alpha_{1}, \beta_{1}}[h(x, t) \varepsilon(x, t)-h(0,0) \varepsilon(x, t)]$, or, using the 2D-BLT

$$
\mathcal{L}_{x t}\left[\left[D_{x t}^{\alpha_{1}, \beta_{1}} h(x, t)\right] \varepsilon(x, t)\right]=u^{\alpha_{1}} s^{\beta_{1}} \mathcal{L}_{x t}[h(x, t) \varepsilon(x, t)]-h(0,0) u^{\alpha_{1}-1} s^{\beta_{1}-1} .
$$

The process repeats every time we do any derivative computation. In fact, assume that we want to perform a second derivative computation of orders $\alpha_{2}$ and $\beta_{2}$. We have the substitution

$$
\begin{gathered}
{\left[D_{x t}^{\alpha_{1}+\alpha_{2}, \beta_{1}+\beta_{2}} h(x, t)\right] \varepsilon(x, t) \Rightarrow} \\
D_{x t}^{\alpha_{1}+\alpha_{2}, \beta_{1}+\beta_{2}}\left[h(x, t) \varepsilon(x, t)-\left(D_{x t}^{\alpha_{2}, \beta_{2}} h(0,0)\right) \varepsilon(x, t)\right]-h(0,0) D_{x t}^{\alpha_{1}+\alpha_{2}-1, \beta_{1}+\beta_{2}-1} \varepsilon(x, t) .
\end{gathered}
$$

The general expression may become hard to write, but in most applications this formula is sufficient. In terms of the 2D-BLT it reads:

$$
\begin{aligned}
& \mathcal{L}_{x t}\left[\left[D_{x t}^{\alpha_{1}+\alpha_{2}, \beta_{1}+\beta_{2}} h(x, t)\right] \varepsilon(x, t)\right]=u^{\alpha_{1}+\alpha_{2}} s^{\beta_{1}+\beta_{2}} \mathcal{L}_{x t}[h(x, t) \varepsilon(x, t)]- \\
& h(0,0) u^{\alpha_{2}-1} s^{\beta_{2}-1}-\left[D_{x t}^{\alpha_{1}, \beta_{1}} h(0,0)\right] u^{\alpha_{1}+\alpha_{2}-1} s^{\beta_{1}+\beta_{2}-1} .
\end{aligned}
$$

In the commensurate case, we can write

$$
\begin{array}{r}
\mathcal{L}_{x t}\left[\left[D_{x t}^{2 \alpha, 2 \beta} h(x, t)\right] \varepsilon(x, t)\right]=u^{2 \alpha} s^{2 \beta} \mathcal{L}_{x t}[h(x, t) \varepsilon(x, t)]-h(0,0) u^{\alpha-1} s^{\beta-1}- \\
{\left[D_{x t}^{\alpha, \beta} h(0,0)\right] u^{2 \alpha-1} s^{2 \beta-1},}
\end{array}
$$

which is the $2 \mathrm{D}$ analog of (34).

\section{Conclusions}

This paper revisited the ULT and BLT, both for the 1D and 2D transforms. The ULT and BLT are studied and compared for the 1D case. The systematic treatment lead to the formulation of the initial-condition theorem in the 1D and 2D transforms using the BLT. The case of fractional-order systems was also included. The LT is often loosely interpreted simply as the ULT. However, the fact is that the BLT has advantages from the mathematical point of view that are somewhat overlooked in the applied sciences due to a priori assumptions for negative values of time. On the other hand, recalling the 2D-LT is also relevant, since it is mainly used in the 2-dim FT in the scope of image processing, while it has been neglected in other areas of mathematics and applied sciences. This is particularly important for the multidimensional case since many results are scattered in the literature. This detailed review of the properties and the comprehensive analysis of the LT constitutes a solid ground to pursuit the future development of methods for the representation, analysis and control 2D dynamical systems.

Author Contributions: These two authors contribute equally to this paper. All authors have read and agreed to the published version of the manuscript.

Funding: This work was partially funded by National Funds through the Foundation for Science and Technology of Portugal, under the projects UIDB/00066/2020.

Conflicts of Interest: The authors declare no conflict of interest. 


\section{Abbreviations}

The following abbreviations are used in this manuscript:

LT Laplace transform

ULT unilateral Laplace transform

BLT bilateral Laplace transform

1DLT uni-dimensional Laplace transform

2DLT two-dimensional Laplace transform

FT Fourier transform

ROC region of convergence

IVT initial value theorem

FVT final value theorem

IC initial-conditions

L Liouville

RL Riemann-Liouville

C Caputo

\section{References}

1. Carson, J.R. Electric circuit theory and the operational calculus. Bell Syst. Tech. J. 1925, 4, 685-761. [CrossRef]

2. Carson, J.R. Electric circuit theory and the operational calculus. Bell Syst. Tech. J. 1926, 5, 336-384. [CrossRef]

3. Dorf, R.; Svoboda, J. Introduction to Electric Circuits, 6th ed.; Wiley: New York, NY, USA, 2003.

4. Oppenheim, A.V.; Willsky, A.S.; Hamid, S. Signals and Systems, 2nd ed.; Prentice-Hall: Upper Saddle River, NJ, USA, 1997.

5. Roberts, M. Signals and Systems: Analysis Using Transform Methods and Matlab, 2nd ed.; McGraw-Hill: New York, NY, USA, 2003.

6. Ortigueira, M.D.; Valério, D. Fractional Signals and Systems; De Gruyter: Berlin, Germany; Boston, MA, USA, 2020.

7. Dorf, R.C.; Bishop, R.H. Modern Control Systems; Pearson: London, UK, 2011.

8. Valério, D.; da Costa, J.S. An Introduction to Fractional Control; Control Engineering, IET: Stevenage, UK, 2012.

9. Feller, W. An Introduction to Probability Theory and Its Applications; John Wiley \& Sons: Hoboken, NJ, USA, 1991.

10. Bulmer, M.G. Principles of Statistics; Courier Corporation: North Chelmsford, MA, USA, 1979.

11. Martino, L. Independent Random Sampling Methods; Springer: Berlin/Heidelberg, Germany, 2018.

12. Uchaikin, V.V.; Zolotarev, V.M. Chance and Stability: Stable Distributions and Their Applications; Walter de Gruyter: Berlin, Germany, 2011.

13. Magin, R.L. Fractional Calculus in Bioengineering; Begell House: Danbury, CT, USA, 2006.

14. Grote, K.H.; Antonsson, E.K. Springer Handbook of Mechanical Engineering; Springer Science \& Business Media: Berlin, Germany, 2009; Volume 10.

15. Henley, E.J.; Kumamoto, H. Reliability Engineering and Risk Assessment; Prentice-Hall: Englewood Cliffs, NJ, USA, 1981; Volume 568.

16. Riley, K.F.; Hobson, M.P.; Bence, S.J. Mathematical Methods for Physics and Engineering: A Comprehensive Guide; Cambridge University Press: Cambridge, UK, 2006.

17. Bird, J. Higher Engineering Mathematics; Routledge: Abingdon, UK, 2007.

18. Heaviside, O. Electrical Papers; Macmillan Co.: London, UK; New York, NY, USA, 1892; Volume 1.

19. Heaviside, O. Electromagnetic Theory; The Electrician Printing and Publishing Co.: London, UK, 1892; Volume 1.

20. Heaviside, O. Electrical Papers; Macmillan Co.: London, UK; New York, NY, USA, 1894; Volume 2.

21. Heaviside, O. Electromagnetic Theory; The Electrician Printing and Publishing Co.: London, UK, 1899; Volume 2.

22. Heaviside, O. Electromagnetic Theory; The Electrician Printing and Publishing Co.: London, UK, 1912 ; Volume 3.

23. Lévy, P. Le calcul Symbolique D'Heaviside; Gauthier-Villars et Cie: Paris, France 1926.

24. Lévy, P. Le calcul symbolique et ses principales applications. In Annales de l'université de Grenoble; University of Grenoble: Grenoble, France, 1945; Volume 21, pp. 41-56.

25. Lützen, J. Heaviside's operational calculus and the attempts to rigorise it. Arch. Hist. Exact Sci. 1979, 21, 161-200. [CrossRef]

26. Flegg, H.G. A survey of the development of operational calculus. Int. J. Mathamat. Educ. Sci. Technol. 1971, 2, 329-335. [CrossRef] 
27. Deakin, M.A.B. Euler's Version of the Laplace Transform. Am. Math. Mon. 1980, 87, 264-269. [CrossRef]

28. Deakin, M.A.B. The development of the Laplace transform, 1737-1937. Arch. Hist. Exact Sci. 1981, 25, 343-390. [CrossRef]

29. Deakin, M.A.B. The development of the Laplace Transform, 1737-1937 II. Poincaré to Doetsch, 1880-1937. Arch. Hist. Exact Sci. 1982, 26, 351-381. [CrossRef]

30. Liouville, J. Memóire sur le calcul des différentielles à indices quelconques. J. De L'École Polytech. Paris 1832, 13, 71-162.

31. Liouville, J. Memóire sur l'usage que l'on peut faire de la formule de Fourier, dans le calcul des différentielles à indices quelconques. J. Für Die Reine Und Angew. Math. (Journal De Crelle) 1835, 13, 219-232.

32. Liouville, J. Note sur une formule pour les différentielles à indices quelconques à l'occasion d'un mémoire de M. Tortolini. J. De Mathématiques Pures Et Appliquées 1855, 20, 115-120.

33. Ortigueira, M.D. Fractional Calculus for Scientists and Engineers; Lecture Notes in Electrical Engineering; Springer: Berlin/Heidelberg, Germany, 2011.

34. Valério, D.; Machado, J.; Kiryakova, V. Some pioneers of the applications of fractional calculus. Fract. Calc. Appl. Anal. 2014, 17. [CrossRef]

35. Carson, J.R. Die Grundgesetze der Ausgleichsvorgänge in elektrischen Stromkreisen. In Elektrische Ausgleichsvorgänge und Operatorenrechnung; Springer: Berlin/Heidelberg, Germany, 1929; pp. 1-11.

36. Van der Pol, B.; Bremmer, H. Operational Calculus: Based on the Two-sided Laplace Integral; Cambridge University Press: Cambridge, UK, 1950.

37. Doetsch, G. Theorie und Anwendungder Laplace-Transformation; Springer: Berlin, Germany, 1937.

38. Doetsch, G.; Nader, W. Introduction to the Theory and Application of the Laplace Transformation; Springer: Berlin, Germany, 1974.

39. Lundberg, K.H.; Miller, H.R.; Trumper, D.L. Initial conditions, generalized functions, and the Laplace transform: Troubles at the origin. IEEE Control Syst. Mag. 2007, 27, 22-35.

40. Ortigueira, M.D.; Coito, F.J. System initial conditions vs. derivative initial conditions. Comput. Math. Appl. 2010, 59, 1782-1789.

41. Ahuja, S.; Arya, R.K. Consistent Initialization of the Laplace Transform. arXiv 2019, arXiv:abs/1909.07813.

42. Paley, R.; Wiener, N. Fourier Transforms in the Complex Domain; American Mathematical Society: Providence, RI, USA, 1934; Volume 19.

43. Widder, D. The Laplace Transform; Karreman Mathematics Research Collection, Princeton Mathematical Series; Princeton University Press: Princeton, NJ, USA, 1941.

44. Dayal, S.; Singh, M.K. An analysis of convergence of Bi-lateral Laplace Transform. Int. J. Math. Its Appl. 2017, 5, 223-229.

45. Ortigueira, M.; Machado, J.T. Which Derivative? Fractal Fract. 2017, 1, 3. [CrossRef]

46. Zemanian, A.H. Distribution Theory and Transform Analysis: An Introduction to Generalized Functions, with Applications; Lecture Notes in Electrical Engineering; Dover Publications: New York, NY, USA, 1987; Volume 84.

47. Bellman, R.; Roth, R. The Laplace Transform; Modern Applied Mathematics Series; World Scientific: Singapore, 1984.

48. Henrici, P. Applied and Computational Complex Analysis; Wiley-Interscience: Hoboken, NJ, USA, 1991; Volume 2.

49. Prudnikov, A.P.; Skòrnik, K.A. Operational Calculus and Related Topics; CRC Press: Boca Raton, FL, USA, 2006.

50. Ditkin, V.A.; Prudnikov, A.P. Operational Calculus in Two Variables and Its Applications; Courier Dover Publications: Mineola, NY, USA, 2017.

51. Gel'fand, I.M.; Shilov, G.E. Generalized Functions. Volume I: Properties and Operations; Academic Press: New York, NY, USA; London, UK, 1964.

52. Ferreira, J. Introduction to the Theory of Distributions; Pitman Monographs and Surveys in Pure and Applied Mathematics; Pitman: London, UK, 1997.

53. Coon, G.A.; Bernstein, D.L. Some Properties of the Double Laplace Transformation. Trans. Am. Math. Soc. 1953, 74, 135-176. [CrossRef]

54. Mittag-Leffler, M. Sur la nouvelle fonction $E_{\alpha}(x)$. C. R. Acad. Sci. 1903, (Ser. II) 137, 554-558.

55. Mittag-Leffler, M. Sur la nouvelle fonction $E_{\alpha}(x)$. Rend. R. Acc. Lincei 1904, (Ser. 5) 13, 3-5.

56. Mittag-Leffler, M. Sur la representation analytique d'une branche uniforme d'une fonction monogene. Acta Math. 1900, 23, 43-62. [CrossRef] 
57. Gorenflo, R.; Kilbas, A.A.; Mainardi, F.; Rogosin, S.V. Mittag-Leffler Functions, Related Topics and Applications; Springer Monographs in Mathematics; Springer: Berlin/Heidelberg, Germany, 2014.

58. Dattoli, G.; Gorska, K.; Horzela, A.; Licciardi, S.; Pidatella, R.M. Comments on the properties of Mittag-Leffler function. Eur. Phys. J. Spec. Top. 2017, 226, 3427-3443. [CrossRef]

59. Machado, J.T. Generalized convolution. Appl. Math. Comput. 2015, 257, 34-39. [CrossRef]

60. Samko, S.; Kilbas, A.; Marichev, O. Fractional Integrals and Derivatives: Theory and Applications; Gordon and Breach Science Publishers: Amsterdam, The Netherlands, 1993.

61. Erfani, S.; Bayan, N. Characterisation of nonlinear and linear time-varying systems by Laplace transformation. Int. J. Syst. Sci. 2013, 44, 1450-1467. [CrossRef]

62. Ortigueira, M.D.; Magin, R.L.; Trujillo, J.J.; Velasco, M.P. A real regularised fractional derivative. Signal Image Vid. Process. 2012, 6, 351-358. [CrossRef]

63. Ortigueira, M.D. Two-sided and regularised Riesz-Feller derivatives. Math. Methods Appl. Sci. 2019. [CrossRef]

(C) 2020 by the authors. Licensee MDPI, Basel, Switzerland. This article is an open access article distributed under the terms and conditions of the Creative Commons Attribution (CC BY) license (http:/ / creativecommons.org/licenses/by/4.0/). 